PROCEEDINGS OF THE

AMERICAN MATHEMATICAL SOCIETY

Volume 140, Number 6, June 2012, Pages 1957-1962

S 0002-9939(2011)11042-6

Article electronically published on September 26, 201

\title{
ON LYAPUNOV EXPONENTS OF CONTINUOUS SCHRÖDINGER COCYCLES OVER IRRATIONAL ROTATIONS
}

\author{
WEN HUANG AND YINGFEI YI
}

(Communicated by Bryna Kra)

\begin{abstract}
In this paper we consider continuous, $\mathrm{SL}(2, \mathbb{R})$-valued, Schrödinger cocycles over irrational rotations. We prove two generic results on the Lyapunov exponents which improve the corresponding ones contained in a paper by Bjerklöv, Damanik and Johnson.
\end{abstract}

\section{INTRODUCTION}

Let $\alpha$ be a fixed irrational number and $A: \mathbb{T} \mapsto \mathrm{SL}(2, \mathbb{R})$ be a continuous map. Then $A$ generates a continuous, $\mathrm{SL}(2, \mathbb{R})$-valued cocycle $\{A(n, \theta)\}$ over the irrational rotations $\theta \mapsto \theta+\alpha$ on $\mathbb{T}=\mathbb{R} / \mathbb{Z}$ (or a continuous, quasi-periodic, $\mathrm{SL}(2, \mathbb{R}$ )-valued cocycle with frequency $\alpha$ ). More precisely, define

$$
A(n, \theta)= \begin{cases}A(\theta+(n-1) \alpha) \ldots A(\theta), & n>0, \\ I d, & n=0, \\ A^{-1}(\theta-n \alpha) \ldots A^{-1}(\theta-\alpha), & n<0 .\end{cases}
$$

It is clear that $\{A(n, \theta)\}$ satisfies the cocycle property:

$$
A(n+m, \theta)=A(n, \theta+m \alpha) A(m, \theta), m, n \in \mathbb{Z}, \theta \in \mathbb{T} .
$$

The cocycle admits a well-defined (maximal) Lyapunov exponent given by

$$
\Lambda(A):=\lim _{n \rightarrow \infty} \frac{1}{n} \int_{\mathbb{T}} \log \|A(n, \theta)\| d \theta=\inf _{n \geq 1} \frac{1}{n} \int_{\mathbb{T}} \log \|A(n, \theta)\| d \theta ;
$$

i.e., the limit exists and is independent of $\theta$. When $\Lambda(A)>0$, the corresponding cocycle is said to be uniformly hyperbolic if

$$
\lim _{n \rightarrow+\infty} \frac{1}{n} \log \|A(n, \theta)\|=\Lambda(A)
$$

uniformly in $\theta$ and to be non-uniformly hyperbolic if otherwise.

Received by the editors December 5, 2010 and, in revised form, January 30, 2011.

2010 Mathematics Subject Classification. Primary 37B55; Secondary 37D25.

Key words and phrases. Lyapunov exponent, Schrödinger cocycles, non-uniform hyperbolicity.

The first author is partially supported by NSFC(10911120388,11071231), Fok Ying Tung Education Foundation and the Fundamental Research Funds for the Central Universities (WK0010000001,WK0010000014).

The second author is partially supported by NSF grant DMS0708331, NSFC Grant 10428101, and a Changjiang Scholarship from Jilin University.

(C)2011 American Mathematical Society Reverts to public domain 28 years from publication 
In this paper, we pay particular attention to continuous, quasi-periodic, $\operatorname{SL}(2, \mathbb{R})$ valued, Schrödinger cocycles with fixed irrational frequency $\alpha$, i.e., a family $\left\{A_{f, E}(n, \theta): E \in \mathbb{R}, f \in C(\mathbb{T})\right\}$ of quasi-periodic, $\mathrm{SL}(2, \mathbb{R})$-valued cocycles with the frequency $\alpha$ which is generated by the continuous, $\mathrm{SL}(2, \mathbb{R})$-valued functions

$$
A_{f, E}(\theta)=\left(\begin{array}{cc}
E-f(\theta) & -1 \\
1 & 0
\end{array}\right) .
$$

Such cocycles are referred to as Schrödinger cocycles because they arise and play important roles in the study of the spectral problem of the discrete quasi-periodic Schrödinger operator,

$$
\left[H_{f} \psi\right](n)=(\Delta+f(\theta+(n-1) \alpha)) \psi(n)=E \psi(n),
$$

where $\Delta \psi(n)=\psi(n+1)+\psi(n-1)$. For simplicity, we denote $\Lambda_{f}(E)=: \Lambda\left(A_{f, E}\right)$, $A_{f}(n, \cdot)=: A_{f, 0}(n, \cdot)$, and $\Lambda_{f}=: \Lambda_{f}(0)$.

Related to the spectral problem especially with respect to the non-existence of an absolutely continuous spectrum, one often considers, for a fixed $f$, a twoparameter family $\left\{A_{\lambda f, E}(n, \theta)\right\}$ of Schrödinger cocycles, and studies the positivity of the Lyapunov exponents $\Lambda_{\lambda f}(E)$ for $\lambda$ sufficiently large. In particular, when $\alpha$ satisfies appropriate Diophantine conditions, for a certain class of smooth $f$, it is known that $\Lambda_{\lambda f}(E)$ is of scale of $\log \lambda$ as $\lambda \gg 1$ uniformly in $E$ (see, e.g., 2, 6, 11, 12, [16]). However, in a recent work of Bjerklöv, Damanik, and Johnson [3] such uniform bounds are shown to be extremely unstable within the class of continuous functions. More precisely, it is shown in 3 that for every countable set $\left\{\lambda_{m}\right\}_{m=1}^{\infty} \subset(0,+\infty)$, there exists a residual set of $f \in C(\mathbb{T})$ for which $\inf _{E \in \mathbb{R}} \Lambda_{\lambda_{m} f}(E)=0$ for each $m \in \mathbb{N}$.

In this paper, we will show that this result can be improved as follows.

Theorem 1. For a residual set of $f \in C(\mathbb{T})$,

$$
\inf _{E \in \mathbb{R}} \Lambda_{\lambda f}(E)=0
$$

for any $\lambda>0$.

For general quasi-periodic, continuous, $\mathrm{SL}(2, R)$-valued cocycles, it is shown in [4] that there is a residual set $\mathcal{R} \subset C(\mathbb{T}, S L(2, \mathbb{R}))$ such that for $A \in \mathcal{R}$, either $A$ is uniformly hyperbolic or $\Lambda(A)=0$ (see [9, 10] for similar results that hold for a generic set of pairs $(\alpha, f)$; see also [1]). The same is also shown to hold for Schrödinger cocycles with $E=0(3,5])$.

Our next result proves the same phenomenon for the parametrized Schrödinger cocycles with $E=0$.

Theorem 2. The set

$$
\left\{f \in C(\mathbb{T}): A_{\lambda f}(n, \cdot) \text { is uniformly hyperbolic or } \Lambda_{\lambda f}=0 \text { for any } \lambda \in(0, \infty)\right\}
$$

is residual.

The rest of this paper is devoted to the proof of Theorems 1 and 2. Our proofs essentially follow the approaches of [3] with necessary modifications. 


\section{Proofs of theorems}

Throughout the rest of the paper, we let $\alpha$ be a fixed irrational number. For a Schrödinger operator $H_{f}$ of the form (1.1) with $\theta \in \mathbb{T}$ and $f \in L^{1}(\mathbb{T})$, it is well known that the spectrum $\sigma\left(H_{f}\right)$ is independent of $\theta \in \mathbb{T}$ almost everywhere, and if $f \in C(\mathbb{T})$, then $\sigma\left(H_{f}\right)$ is completely independent of $\theta$. Uniform and non-uniform hyperbolicities of the corresponding (measurable) Schrödinger cocycles $A_{f, E}(n, \cdot)$ can be defined similarly to the continuous case.

As in [3], the following result will play an important role in the proofs of the theorems.

Theorem 2.1. Suppose $f: \mathbb{T} \mapsto \mathbb{R}$ is of the form

$$
f(\theta)=\sum_{m=1}^{M} f_{m} \chi_{\left[\beta_{m-1}, \beta_{m}\right)}(\theta),
$$

where $0=\beta_{0}<\beta_{1}<\cdots<\beta_{M}=1$ are rational numbers and $f_{1}, \ldots, f_{M}$ are real. Then $\sigma\left(H_{f}\right)=\left\{E: \Lambda_{f}(E)=0\right\}$.

Proof. See [7, 8].

A crucial step in proving the above result is to show that for any $f$ having the form (2.1),

$$
\lim _{n \rightarrow \infty} \frac{1}{n} \log \left\|A_{f, E}(n, \theta)\right\|=\Lambda_{f}(E)
$$

for every $E \in \mathbb{R}$ uniformly in $\theta \in \mathbb{T}([7,8,14])$. The result then follows from the following.

Theorem 2.2. For any $f \in L^{1}(\mathbb{T})$,

$$
\sigma\left(H_{f}\right)=\left\{E: \Lambda_{f}(E)=0 \text { or } A_{f, E}(n, \cdot) \text { is non-uniformly hyperbolic }\right\} .
$$

Proof. See [15, 13].

Lemma 2.3. For any non-empty compact subset $K \subseteq(0,+\infty)$, the set

$$
M_{K, 0}:=\left\{f \in C(\mathbb{T}): \inf _{E \in \mathbb{R}} \Lambda_{\lambda f}(E)=0 \text { for any } \lambda \in K\right\}
$$

is residual in $C(\mathbb{T})$.

Proof. Let $K \subseteq(0,+\infty)$ be a non-empty compact subset. We consider the family of sets

$$
M_{K, \delta}=\left\{f \in C(\mathbb{T}): \forall \lambda \in K \exists E_{\lambda} \in \mathbb{R} \text { such that } \Lambda_{\lambda f}\left(E_{\lambda}\right)<\delta\right\}, \delta>0 .
$$

We will show that each $M_{K, \delta}$ is open and dense, and hence $M_{K, 0}=\bigcap_{\delta>0} M_{K, \delta}$ is residual.

First we show that $M_{K, \delta}$ is open, i.e., $C(\mathbb{T}) \backslash M_{K, \delta}$ is closed. Let $\left\{f_{n}\right\} \subset C(\mathbb{T}) \backslash$ $M_{K, \delta}, f \in C(\mathbb{T})$ be such that $\left\|f_{n}-f\right\|_{\infty} \rightarrow 0$. Then for each $n \in \mathbb{N}$ there exists a $\lambda_{n} \in K$ with $\Lambda_{\lambda_{n} f_{n}}(E) \geq \delta$ for all $E \in \mathbb{R}$. Since $K$ is compact, there exists a subsequence $\left\{n_{1}<n_{2}<\cdots\right\} \subseteq \mathbb{N}$ such that $\lim _{i \rightarrow \infty} \lambda_{n_{i}}=\lambda_{0}$ for some $\lambda_{0} \in K$. It follows from the upper-semicontinuity of Lyapunov exponents $\Lambda_{\lambda f}(E)$ in $\lambda$ that

$$
\Lambda_{\lambda_{0} f}(E) \geq \limsup _{i \rightarrow \infty} \Lambda_{\lambda_{n_{i}} f_{n_{i}}}(E) \geq \delta
$$

for any $E \in \mathbb{R}$. Hence $f \in C(\mathbb{T}) \backslash M_{K, \delta}$. This shows that $C(\mathbb{T}) \backslash M_{K, \delta}$ is closed. 
Next we show that $M_{K, \delta}$ is dense. Let $\epsilon>0$ and $g \in C(\mathbb{T})$ be given. In the $\frac{\epsilon}{2}$-neighborhood of $g$ with respect to the $L^{\infty}$ topology, we choose a step function $s$ of the form (2.1); i.e., $s$ has finitely many points of discontinuity, all of which are rational, and the jumps of $s$ are bounded by $\frac{\epsilon}{2}$. It then follows from Theorem 2.1 that for any $\lambda \in K, \Lambda_{\lambda s}$ vanishes on the spectrum $\sigma\left(H_{\lambda s}\right)$ of $H_{\lambda s}$; i.e., there exists an $E_{\lambda} \in \sigma\left(H_{\alpha, \lambda s}\right)$ such that $\Lambda_{\lambda s}\left(E_{\lambda}\right)=0$. By the upper-semicontinuity of Lyapunov exponents, there exists a $\delta_{\lambda}>0$, for each $\lambda \in K$, such that $\Lambda_{u s}\left(E_{\lambda}\right)<\delta$ for any $u \in B\left(\lambda, \delta_{\lambda}\right):=\left\{t \in \mathbb{R}:|t-\lambda|<\delta_{\lambda}\right\}$. As $K$ is compact, there exist $u_{1}, \ldots, u_{\ell} \in K$ such that $K \subseteq \bigcup_{i=1}^{\ell} B\left(u_{i}, \frac{\delta_{u_{i}}}{2}\right)$. Then

$$
\Lambda_{\lambda s}\left(E_{u_{i}}\right)<\delta \text { for all } 1 \leq i \leq \ell \text { and } \lambda \in B\left(u_{i}, \delta_{u_{i}}\right) \cap K .
$$

Let $\left\{f_{n}\right\} \subset C(\mathbb{T})$ be such that $\int_{\mathbb{T}}\left|s(\theta)-f_{n}(\theta)\right| d \theta<\frac{1}{n}$ and $\left\|s-f_{n}\right\|_{\infty}<\frac{\epsilon}{2}$ for all $n \in \mathbb{N}$. We claim that there exists an $n_{*} \in \mathbb{N}$ such that $\Lambda_{\lambda f_{n_{*}}}\left(E_{u_{i}}\right)<\delta$ for all $1 \leq i \leq \ell$ and $\lambda \in B\left(u_{i}, \frac{\delta_{u_{i}}}{2}\right) \cap K$; i.e., $f=: f_{n_{*}}$ has the desired properties that $f \in M_{K, \delta}$ and $\|f-g\|_{\infty}<\epsilon$.

If the claim is not true, then for each $n \in \mathbb{N}$ there exist $i_{n} \in\{1,2, \ldots, \ell\}$ and $\lambda_{n} \in B\left(u_{i_{n}}, \frac{\delta_{u_{i_{n}}}}{2}\right) \cap K$ such that $\Lambda_{\lambda_{n} f_{n}}\left(E_{u_{i_{n}}}\right) \geq \delta$. Without loss of generality, we assume that $\lim _{n \rightarrow \infty} \lambda_{n}=\lambda_{0}$ for some $\lambda_{0} \in K$ and $i_{n} \equiv i_{0} \in\{1,2, \ldots, \ell\}$ for all $n \in \mathbb{N}$. It is clear that $\lambda_{0} \in B\left(u_{i_{0}}, \delta_{u_{i_{0}}}\right) \cap K$ and $\lim _{n \rightarrow \infty} \int_{\mathbb{T}}\left|\lambda_{0} s(\theta)-\lambda_{n} f_{n}(\theta)\right| d \theta=0$. Hence by the upper semi-continuity of Lyapunov exponents, we have

$$
\delta>\Lambda_{\lambda_{0} s}\left(E_{u_{i_{0}}}\right) \geq \limsup _{n \rightarrow \infty} \Lambda_{\lambda_{n} f_{n}}\left(E_{u_{i_{0}}}\right) \geq \delta,
$$

a contradiction.

Proof of Theorem 1. Let $K_{n}=\left[\frac{1}{n}, n\right], n \in \mathbb{N}$. Then by Lemma 2.3,

$$
\left\{f \in C(\mathbb{T}): \inf _{E \in \mathbb{R}} \Lambda_{\lambda f}(E)=0 \text { for any } \lambda>0\right\}=\bigcap_{n=1}^{\infty} M_{K_{n}, 0}
$$

is residual.

Proof of Theorem 2. It is sufficient to show that for any non-empty compact set $K \subseteq(0, \infty)$, the set

$$
N_{K}=\left\{f \in C(\mathbb{T}): \exists \lambda \in K \text { s.t. } A_{\lambda f}(n, \cdot) \text { is non-uniformly hyperbolic }\right\}
$$

is a meagre set, i.e., a countable union of nowhere-dense sets. This will follow once we prove that

$$
\begin{aligned}
N_{K, \gamma}=\left\{f \in C(\mathbb{T}): \exists \lambda \in K \text { s.t. } A_{\lambda f}(n, \cdot)\right. \\
\text { is non-uniformly hyperbolic and } \left.\Lambda_{\lambda f} \geq \gamma\right\}
\end{aligned}
$$

is nowhere dense for every $\gamma>0$.

Let $\gamma>0$ be given. We first show that $N_{K, \gamma}$ is closed. Let $\left\{f_{i}\right\} \subset N_{K, \gamma}$ and $f_{0} \in C(\mathbb{T})$ be such that $\lim _{i \rightarrow \infty}\left\|f_{i}-f_{0}\right\|_{\infty}=0$. Then for each $i \in \mathbb{N}$, there exists a $\lambda_{i} \in K$ such that $A_{\lambda_{i} f_{i}}(n, \cdot)$ is non-uniformly hyperbolic and $\Lambda_{\lambda_{i} f_{i}} \geq \gamma$. Without loss of generality, we assume that $\lim _{i \rightarrow \infty} \lambda_{i}=\lambda_{0}$ for some $\lambda_{0} \in K$. Then $\lim _{i \rightarrow \infty}\left\|\lambda_{i} f_{i}-\lambda_{0} f_{0}\right\|_{\infty}=0$, and hence $\Lambda_{\lambda_{0} f_{0}} \geq \lim \sup _{i \rightarrow \infty} \Lambda_{\lambda_{i} f_{i}} \geq \gamma$ according to the upper semi-continuity of Lyapunov exponents. Since uniform hyperbolicity is an open property, $A_{\lambda_{0} f_{0}}(n, \cdot)$ is non-uniformly hyperbolic. This shows that $f_{0} \in N_{K, \gamma}$. Hence $N_{K, \gamma}$ is closed. 
Next we show that $N_{K, \gamma}$ has no interior. This amounts to showing that for any given $f \in N_{K, \gamma}$ and $\epsilon>0$ there exists a function $g \in C(\mathbb{T})$ such that $\|f-g\|_{\infty}<\epsilon$ and $g \notin N_{K, \gamma}$. For the given $f \in N_{K, \gamma}$, we let $\lambda_{*} \in K$ be such that $A_{\lambda_{*} f}(n, \cdot)$ is non-uniformly hyperbolic and $\Lambda_{\lambda_{*} f} \geq \gamma$. Also let $\left\{s_{m}\right\}$ be a sequence of step functions of the form (2.1) in the $\frac{\epsilon}{4}$-neighborhood of $f$ that converge to $f$ in the $L^{\infty}$ topology. Then for each $\theta \in \mathbb{T}$, the operators $H_{m}=\Delta+\lambda_{*} s_{m}(\cdot \alpha+\theta)$ converge strongly to $H=\triangle+\lambda_{*} f(\cdot \alpha+\theta)$. Since $A_{\lambda_{*} f}(n, \cdot)$ is non-uniformly hyperbolic, we have by Theorem 2.2 that $0 \in \sigma(H)$. By the strong convergence of $H_{m}$, we also have a sequence $\left\{E_{m}\right\} \subset \sigma\left(H_{m}\right)$ such that $E_{m} \rightarrow 0$. Now let $m \gg 1$ be fixed such that $\left|E_{m}\right|<\frac{\epsilon}{4}$. Then $s=s_{m}-E_{m}$ is a step function of the form (2.1) in the $\frac{\epsilon}{2}$-neighborhood of $f$ such that 0 belongs to the spectrum of $\bar{H}=\Delta+\lambda_{*} s(\cdot \alpha+\theta)$. It follows from Theorem 2.1 that $\Lambda_{\lambda_{*} s}=0$. Now consider a sequence of continuous functions $\left\{g_{i}\right\} \subset C(\mathbb{T})$ with $\int_{\mathbb{T}}\left|s(\theta)-g_{i}(\theta)\right| d \theta<\frac{1}{i}$ and $\left\|s-g_{i}\right\|_{\infty}<\frac{\epsilon}{2}$ for all $i \in \mathbb{N}$. We have by the upper semi-continuity of Lyapunov exponents that

$$
0=\Lambda_{\lambda_{*} s} \geq \lim _{i \rightarrow \infty} \Lambda_{\lambda_{*} g_{i}} .
$$

Hence we can choose a $k \in \mathbb{N}$ such that the function $g=g_{k}$ has the desired properties that $\Lambda_{\lambda_{*} g}<\gamma$ and $\|f-g\|_{\infty}<\epsilon$.

\section{REFERENCES}

1. A. Avila and J. Bochi, A uniform dichotomy for generic $\mathrm{SL}(2, \mathbb{R})$ cocycles over a minimal base, Bull. Soc. Math. France 135 (2007), no. 3, 407-417. MR2430187 (2009e:37013)

2. K. Bjerklöv, Positive Lyapunov exponent and minimality for a class of 1-d quasi-periodic Schrödinger equations, Ergodic Theory Dynam. Systems 25 (2005), no. 4, 1015-1045. MR2158395 (2007c:47035)

3. K. Bjerklöv, D. Damanik and R.A. Johnson, Lyapunov exponents of continuous Schrödinger cocycles over irrational rotations, Ann. Mat. Pura Appl. 187 (2008), no. 1, 1-6. MR 2346005 (2008j:37036)

4. J. Bochi, Genericity of zero Lyapunov exponents, Ergodic Theory Dynam. Systems 22 (2002), 1667-1696. MR1944399 (2003m:37035)

5. J. Bochi and M. Viana, The Lyapunov exponents of generic volume-preserving and symplectic maps, Ann. of Math. (2) 161 (2005), 1423-1485. MR2180404(2006j:37060)

6. J. Bourgain and M. Goldstein, On nonperturbative localization with quasi-periodic potential, Ann. of Math. (2) 152 (2000), 835-879. MR1815703 (2002h:39028)

7. D. Damanik and D. Lenz, A condition of Boshernitzan and uniform convergence in the Multiplicative Ergodic Theorem, Duke Math. J. 133 (2006), 95-123. MR2219271(2007a:37004)

8. D. Damanik and D. Lenz, Zero-measure Cantor spectrum for Schrödinger operators with low-complexity potentials, J. Math. Pures Appl. (9) 85 (2006), no. 5, 671-686. MR2229664 (2007a:47034)

9. R. Fabbri, On the Lyapunov exponent and exponential dichotomy for the quasi-periodic Schrödinger operator, Boll. Unione Mat. Ital. Sez. B Artic. Ric. Mat. (8) 5 (2002), no. 1, 149-161. MR.1881929 (2002m:34125)

10. R. Fabbri and R. Johnson, On the Lyapounov exponent of certain $\operatorname{SL}(2, \mathbf{R})$-valued cocycles, Differential Equations Dynam. Systems 7 (1999), no. 3, 349-370. MR1861078 (2002h:37041)

11. M. Goldstein and W. Schlag, Hölder continuity of the integrated density of states for quasiperiodic Schrödinger equations and averages of shifts of subharmonic functions, Ann. of Math. (2) 154 (2001), no. 1, 155-203. MR1847592(2002h:82055)

12. M. Herman, Une méthode pour minorer les exposants de Lyapunov et quelques exemples montrant le caractère local d'un théorème d'Arnold et de Moser sur le tore de dimension 2, Comment. Math. Helv. 58 (1983), no. 3, 453-502. MR727713 (85g:58057)

13. R. Johnson, Exponential dichotomy, rotation number, and linear differential operators with bounded coefficients, J. Differential Equations 61 (1986), no. 1, 54-78. MR818861|(87e:47065) 
14. D. Lenz, Uniform ergodic theorems on subshifts over a finite alphabet, Ergodic Theory Dynam. Systems 22 (2002), no. 1, 245-255. MR1889573 (2003j:37008)

15. D. Lenz, Singular spectrum of Lebesgue measure zero for one-dimensional quasicrystals, Comm. Math. Phys. 227 (2002), 119-130. MR1903841 (2003k:47043)

16. E. Sorets and T. Spencer, Positive Lyapunov exponents for Schrödinger operators with quasiperiodic potentials, Comm. Math. Phys. 142 (1991), 543-566. MR1138050 (93b:81058)

Wu Wen-Tsun Key Laboratory of Mathematics, UStC, Chinese Academy of Sciences, Hefei Anhui 230026, People's Republic of China

E-mail address: wenh@mail.ustc.edu.cn

School of Mathematics, Jilin University, Changchun, 130012, People's Republic of China - And - School of Mathematics, Georgia Institute of Technology, Atlanta, Georgia 30332

E-mail address: yi@math.gatech.edu 